\title{
GENERATING THE CUTTING PATH OF THE SHEET OF METAL WITH VISIBLE PHOTOGRAMETRIC DIFFERENCE OF THE TEMPLATE AND THE OBJECT FOR INDUSTRIAL ROBOT WITH THE VISION SYSTEM
}

\author{
A. Annusewicz ${ }^{*}$, J. Zwierzchowski**
}

\begin{abstract}
The subject of the paper is a system which consists of an industrial robot with a vision system. This system is used to generate a cutting path of the sheet of metal, which is rectangular. The robot is controlled by a computer application that together with a digital camera creates a vision system. The project developed an algorithm that calculates the coordinates of the vertices of the searched object on the basis of straight lines detected by the vision system. The windows application was written in $C++$. The image processing algorithms were based on OpenCV libraries. The main purpose of the robot is to communicate with the application, receive commands and implement a set trajectory, which depends on the location of the object. The program for industrial robot was written in $A S$.
\end{abstract}

Keywords: vision system, image processing, industrial robot.

\section{Introduction}

One of the most important senses for human is the sense of sight. It allows collecting information about the environment and makes it possible to make a decision. Similarly, in automatics, vision systems significantly increase the scope of obtained information and allow for greater autonomy of machines in industry. They also provide the ability to automate decision-making processes. Vision systems are widely used in industry, including quality control processes.

The paper proposes a solution using a vision system that works with an industrial robot. This approach allows generating the cutting path of the sheet metal with a large photogrammetric difference of the object and template, which is located anywhere on the work table.

\section{Description of the system for generating the path}

The test stand consists of the Kawasaki industrial robot with a digital camera (Figure 2) and a computer with a window application that manages the robot's work. The basic task of an industrial robot is to generate the path of cutting the object in the shape of a rectangle or square.

The system operation process can be divided into several stages. The general algorithm is shown in the diagram in Figure 1. After starting the robot takes the base position. It is a point saved in the program, selected so that the camera can see the whole work table and is set parallel to it. The next step is the analysis of the workspace. Based on the camera image, the application determines the exact location of the object, then communicates with the robot and sends the coordinates of the vertices to it. The last stage

mgr inż. Anna Annusewicz: Department of Automation and Robotics, Faculty of Mechatronics and Mechanical Engineering, Kielce University of Technology, al. Tysiąclecia Państwa Polskiego 7;

25-314 Kielce; PL, aannusewicz@tu.kielce.pl

** dr inż. Jarosław Zwierzchowski: Department of Automation and Robotics, Faculty of Mechatronics and Mechanical Engineering, Kielce University of Technology, al. Tysiąclecia Państwa Polskiego 7; 25-314 Kielce; PL, j.zwierzchowski@tu.kielce.pl 
is to implement the set trajectory determined by the vertices of the object. After receiving the command, the robot moves in a linear motion to each point in succession and cuts the shape of the object. After performing the task, the robot returns to the base position.

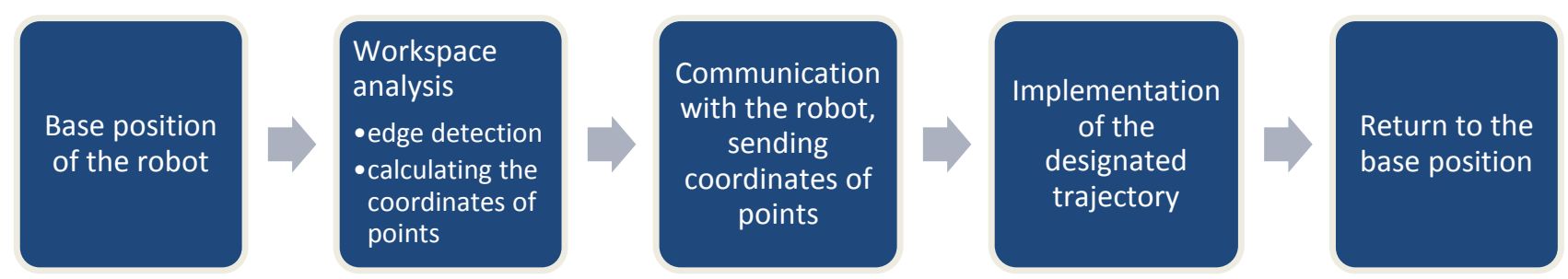

Fig. 1: The scheme of the system for generating the path.

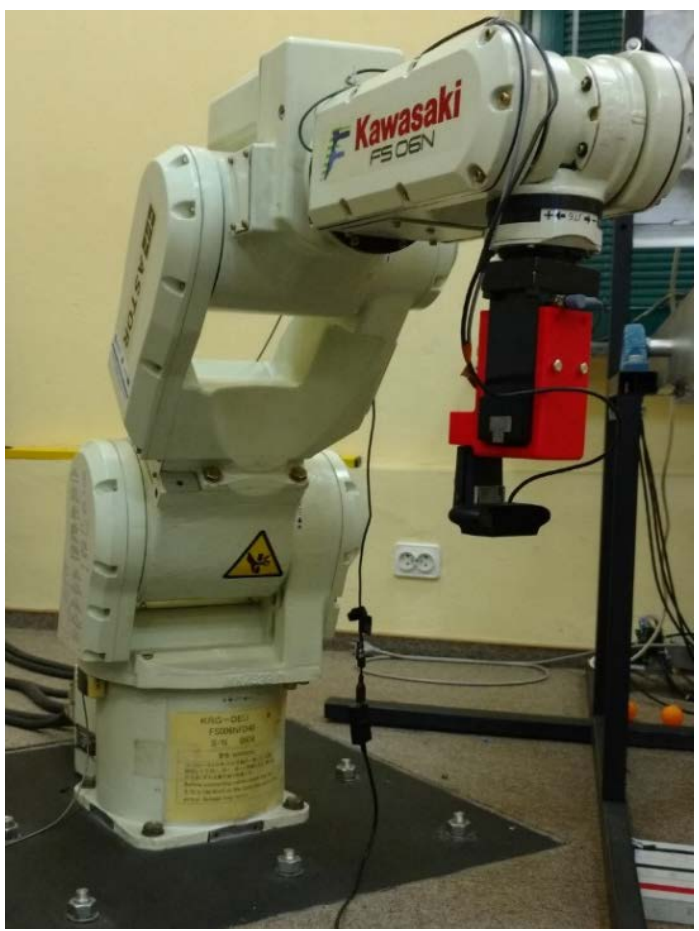

Fig. 2: Robot Kawasaki with digital camera.

\section{Vision system}

The vision system has an important function in the path generation process. It allows finding an object that is anywhere on the work table within the range of the camera.

The first stage in the design of the vision system was to calibrate the digital camera, which allowed converting the coordinates of points from the image to the coordinates of the world system. The calibration was made from the base position of the robot using a checker board. The camera took several photos at different angles to obtain different orientation of the board. The process of parameter selection was performed in the Matlab program using the Camera Calibrator additive.

\subsection{Image processing}

The task of the vision system is to detect the straight edges of the object and find the coordinates of its vertices. In order to detect straight edges in the image, the application uses appropriate filters and transformations. The image from the camera is processed using basic functions from the $\mathrm{C}++$ OpenCV library.

Figure 3 presents the subsequent stages of image processing, the aim of which is to determine the coordinates of the vertices of the object. The first step is to change the color image to a grayscale image and to remove the "pepper and salt" noise with a median filter in order to improve the image quality. The 
next important step is the detection of the edge. The Canny transform was used for this purpose. Before edge detection, the Gauss filter was used, because the Canny operator is sensitive to noise. The Canny edge detector uses the Sobel transform, and the final stage is thresholding with hysteresis. There are two thresholds: low and high, which affect the final selection of pixels belonging to the edge.
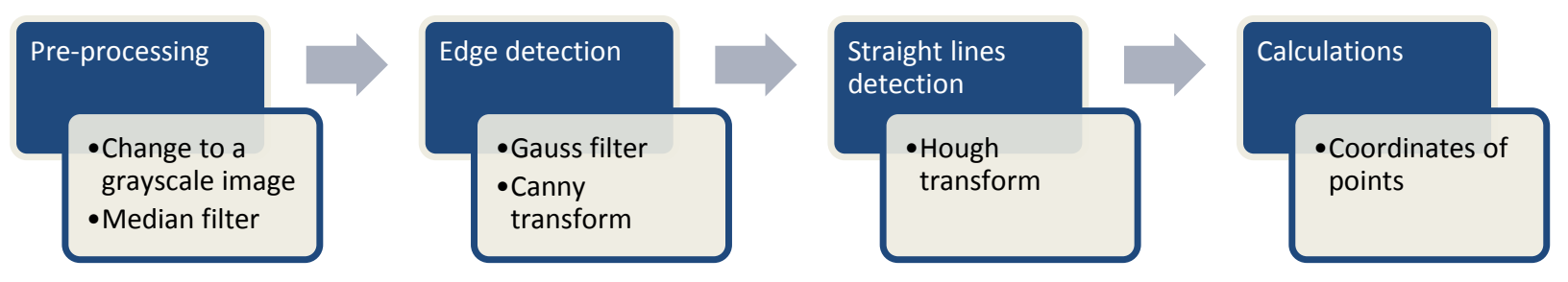

Fig. 3: The image processing scheme.

Using the processed image, it is possible to apply the probabilistic Hough line transform, which determines the straight lines, located on the sides of the object. Figure 4 shows the image from the camera on which the detected straight lines were drawn. The quality of detecting straight lines in the image by the Hough transform depends on the parameters such as the line detection threshold, the minimum number of points forming the line and the acceptable maximum difference between the two points making up the line. The parameters were selected so that only the lines on the sides of the object with the minimum length similar to the length of the sides in the image were detected. The quality of the obtained image from the camera depends on the focus and lighting and has a significant impact on finding the appropriate lines. Due to the fact that the transform sometimes detects several overlapping lines on one side, an algorithm that discards unnecessary straight lines has been implemented. As can be seen in Figure 4, the lines do not completely cover the sides of the object, due to the process of edge detection with the Canny transform, which rounds corners. For this reason, the coordinates of the vertices are unknown at this stage.

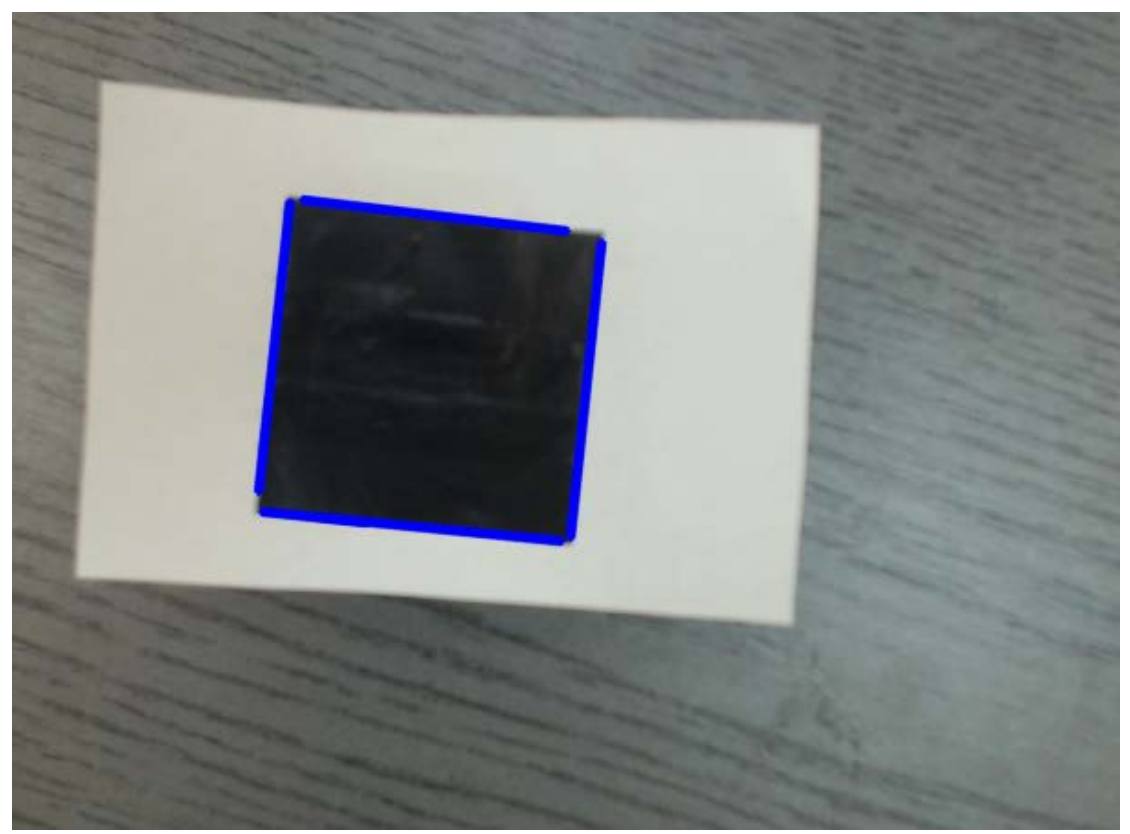

Fig. 4: The result of the Hough algorithm that detects the straight edges of the object in the camera image.

\subsection{An algorithm for determining the coordinates of points}

In order to determine the exact coordinates of the object's vertices, the author developed an algorithm, which is shown in Fig.5. 
Calculation of equations of detected lines

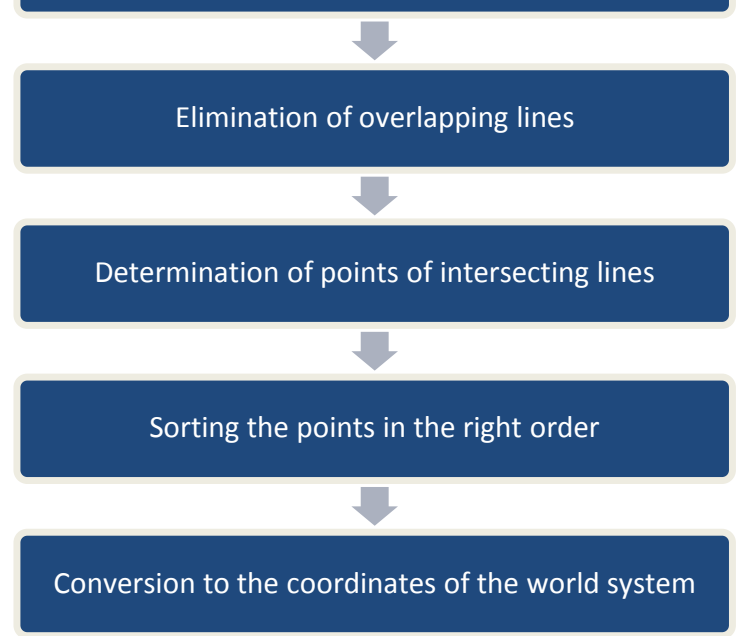

Fig. 5: Simplified algorithm for determining coordinates of points.

The first step is to calculate equations of lines. Due to the possibility of similar straight lines, one straight line representing one side of the object should be selected. At the beginning, the lines are grouped according to line's parameters, in such a way that the lines found on one side belong to the same group. Then there is a choice of the longest straight line from each group. If four straight lines have been selected, it is possible to designate their intersection points that correspond to the vertices of the object in the image. An important aspect for generating the correct path is to set the points in the correct order so that the robot can move around the perimeter of the object. The final stage of the algorithm is to convert coordinates of vertices in the image to coordinates in the world system and send a string type command with calculated coordinates to an industrial robot.

\section{Conclusions}

The use of a vision system for the path generation task for an industrial robot increases the versatility of the system and allows the location of rectangular objects to be detected in the camera area.

As part of the proposed project, a PC application has been developed that is integrated with the Kawasaki industrial robot. In addition, the author wrote a program for a robot in AS language, which allows to read the received commands and is responsible for the robot's movement. The developed window application allows sending precise coordinates of points determining the path of sheet metal cutting.

\section{References}

Canny, J. (1986) A computational approach to edge detection. IEEE Transactions, Pattern Analysis and Machine Intelligence, 6, pp. 679-698.

Czechowicz, A., Mikrut, S. (2006) Analiza przydatności algorytmów detekcji krawędzi w zastosowaniach fotogrametrii bliskiego zasięgu. Archiwum Fotogrametrii, Kartografii i Teledetekcji, 16, pp. 135-146.

Duda, R., O., Hart, P., E. (1972) Use of the Hough transformation to detect lines and curves in pictures. Communications of the ACM, 15, pp. 11-15.

Moeslund, T., B. (2008) Image and Video Processing.

Kawasaki Heavy Industries, Ltd. (2007) Kontroler serii D, Programowanie w języku AS, 90209-1017DED - PL. 